\title{
EchoGéo
}

\section{Les stratégies spatiales de la population haïtienne à Miami}

Cédric Audebert

\section{OpenEdition}

Journals

Édition électronique

URL : https://journals.openedition.org/echogeo/1615

DOI : 10.4000/echogeo.1615

ISSN : 1963-1197

Éditeur

Pôle de recherche pour l'organisation et la diffusion de l'information géographique (CNRS UMR 8586)

Référence électronique

Cédric Audebert, «Les stratégies spatiales de la population haïtienne à Miami », EchoGéo [En ligne], 2 | 2007, mis en ligne le 22 février 2008, consulté le 31 juillet 2021. URL : http://journals.openedition.org/ echogeo/1615; DOI : https://doi.org/10.4000/echogeo.1615

Ce document a été généré automatiquement le 31 juillet 2021.

EchoGéo est mis à disposition selon les termes de la licence Creative Commons Attribution - Pas d'Utilisation Commerciale - Pas de Modification 4.0 International (CC BY-NC-ND) 


\title{
Les stratégies spatiales de la population haïtienne à Miami
}

\author{
Cédric Audebert
}

Introduction

Par son rôle d'interface et les enjeux mobilitaires dont elle est le théâtre (photos 1 et2), Miami constitue un terrain d'étude idéal de l'interdépendance et de la rencontre entre sociétés « développées » et sociétés « en développement » dans les Amériques. A partir d'une analyse de la compétition spatiale entre les communautés ethnicisées et entre les activités qu'elles génèrent, l'hypothèse est avancée que la structuration sociale de Miami fondée sur la différenciation et la hiérarchisation entre les populations de l'aire métropolitaine produit de la distance dans les rapports sociaux et de l'inégalité dans l'accessibilité aux ressources urbaines. Il en résulte des modes de territorialisation variés, complémentaires et concurrents donnant une réelle épaisseur à la «métropoleinterface ». Dans ce cadre, l'immigration haïtienne est appréhendée dans une perspective socio-spatiale novatrice, où l'insertion des nouveaux venus s'opère dans le cadre de logiques communautaires qui s'incarnent dans le territoire. Cette approche complète tout en la renouvelant une recherche sur la diaspora haïtienne encore majoritairement focalisée sur New York et le Québec (Catanese, 1999; Glick Schiller, Fouron, 1999 ; Mittelberg, Waters, 1992 ; Laguerre, 1984). Elle introduit en outre une dimension socio-géographique nouvelle par rapport aux travaux essentiellement sociologiques et anthropologiques (Portes et Stepick, 1993 ; Grenier et Stepick, 1992) ou démo-géographiques (Boswell, 1994, 1995) sur Miami et ses immigrés. 
Photo 1 - Brickell, quartier des banques à Miami

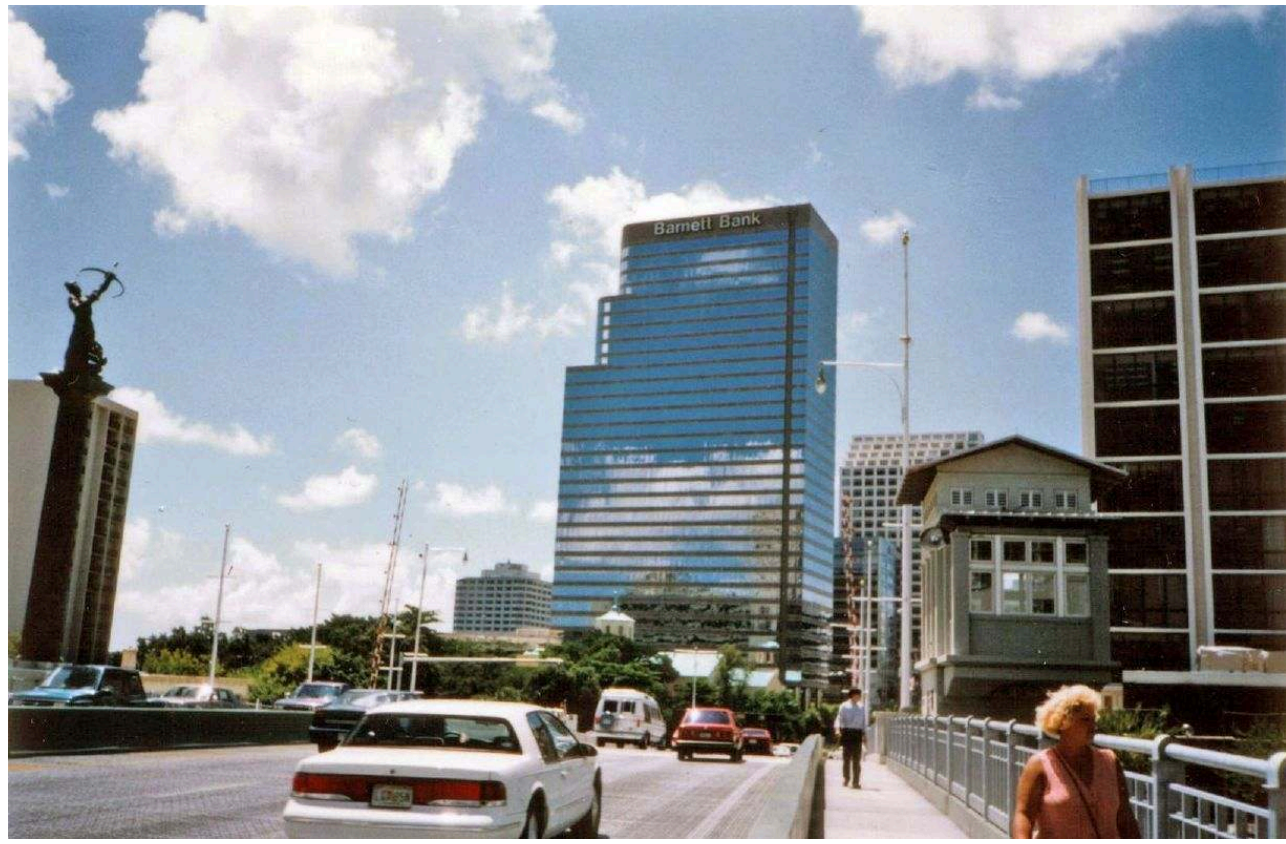

Miami, capitale financière et logistique de la Caraïbe

Photo 2 -Le port de Miami, interface logistique entre les Etats-Unis et la Caraïbe

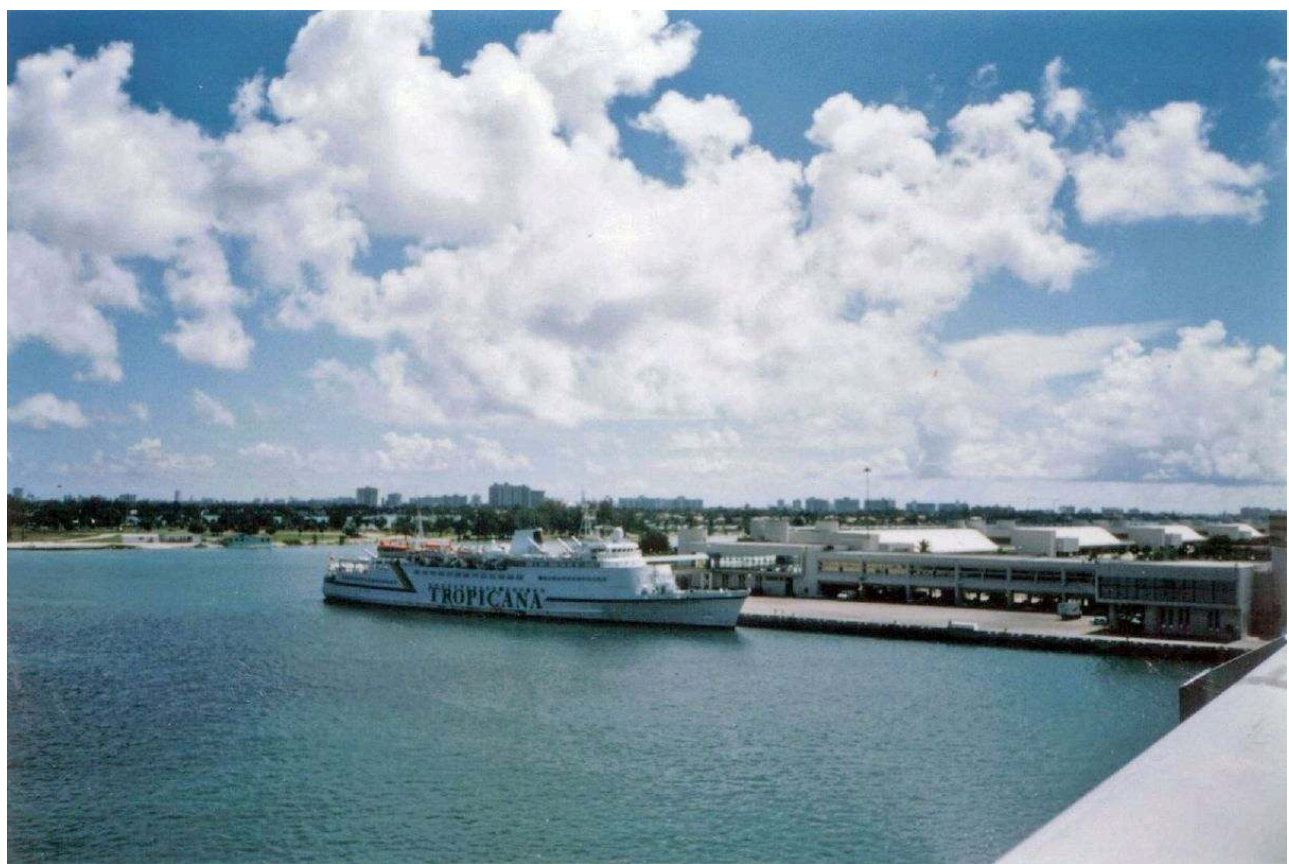

2 La réflexion est fondée sur l'idée d'une double interaction entre les enjeux sociaux et les processus spatiaux, et entre la population immigrée et la société d'installation. Cette double interaction suscite trois questionnements. Le premier est relatif aux enjeux de l'insertion sociale et spatiale de la population d'origine haïtienne à Miami. Le second porte sur la façon dont le rapport d'altérité entre la population haïtienne et la société d'installation s'établit, se reproduit et est instrumentalisé. Une ultime interrogation est liée à la fonction de l'espace en tant que produit et producteur, acteur et enjeu du 
processus de catégorisation et de structuration sociale des populations dans la ville. Les incidences de cette distance produite dans les rapports sociaux sur les processus de territorialisation de la population d'origine haïtienne à Miami sont analysées, avant de montrer comment cet espace où les originaires d'Haïti se sont installés - et qu'ils se sont appropriés - est utilisé et valorisé par eux en vue de réaliser leur intégration dans la société réceptrice.

1. De la concentration à la diffusion spatiale : la constitution d'un espace urbain haïtien à Miami

3 L'analyse du contexte de la genèse historique de Miami - ville à peine centenaire - met en exergue la persistance d'une segmentation sociale et spatiale héritée de l'époque de la ségrégation institutionnalisée (avant 1968) et reproduite depuis quarante ans par les néo-immigrants. L'insertion résidentielle originelle des Haïtiens à Miami, espace métropolitain organisé historiquement sur le mode de la ségrégation sociale et « ethnoraciale », illustre le schéma général d'installation des immigrants récents pauvres dans les quartiers centraux paupérisés et dégradés de nombreuses grandes villes à travers le monde.

4 Cette forme d'insertion spatiale dans les secteurs a priori répulsifs révèle les options résidentielles limitées de nouveaux venus inscrits au bas de l'échelle socio-économique de la société d'accueil. Dans le cadre d'une société états-unienne organisée sur la base de groupes «ethniques", la dimension "ethno-raciale» de la ségrégation urbaine prend cependant une forme exacerbée au point d'exercer une influence aussi remarquable que sa dimension socio-économique. Ainsi, les segmentations sectorielle et auréolaire de Miami portent toutes deux la marque de cette division ethnique de l'espace.

5 Néanmoins, la spécificité du contexte urbain de Miami liée à sa population majoritairement immigrée et latino-américaine tend à remettre partiellement en cause la pertinence des modèles de l'école de Chicago : la «majorité » anglo-saxonne - dont les immigrés sont censés acquérir progressivement les valeurs au cours du processus d'assimilation - apparaît en effet minoritaire tant en termes de poids démographique que d'occupation de l'espace. Dans cette ville de minorités, la minorité traditionnelle noire états-unienne est restée en marge du processus d'intégration sociale et spatiale au profit des «nouvelles » minorités (hispano-caraïbéennes) récemment apparues sur la scène métropolitaine.

6 Dans cet environnement urbain préfigurant le devenir de nombreuses métropoles aux Etats-Unis, l'expérience haïtienne des années 1970 et du début des années 1980 suggère la prééminence des facteurs ethno-culturels et socio-économiques dans l'insertion spatiale des immigrants. Pauvres, noirs et non hispanophones, les Haïtiens se sont installés dans les seuls quartiers qui leur étaient accessibles, ceux du centre-nord de l'aire métropolitaine. Quartier ancien en pleine transition ethno-résidentielle et aux loyers abordables, le périmètre d'Edison-Little River est en particulier devenu dès la deuxième moitié des années 1970 le principal secteur de concentration haïtienne de Floride, au point d'être rebaptisé « Little Haiti »

\section{L'aire métropolitaine de Miami}

7 La dynamique spatiale de la population haïtienne dans l'aire métropolitaine de Miami est le produit de forces tant complémentaires que contradictoires: la ségrégation ethnique et sociale qui, conjuguée à l'immigration massive, a fait de la 
concentration la forme principale de l'insertion spatiale des Haïtiens dans la ville; l'insertion sociale et économique des immigrés qui a élargi l'éventail de leurs options résidentielles; la reproduction par les ménages en terre d'immigration de stratégies de solidarités à l'œuvre dans le pays d'origine permettant de pallier l'insertion des individus au bas de l'échelle sociale et de contourner une politique migratoire intransigeante (Audebert, 2004; Stepick, 1992, 1998). Les incidences de la politique fédérale d'immigration apparaissent elles-mêmes contrastées, occasionnant le repli sur soi des nouveaux venus dans l'enclave lorsqu'elle était défavorable, ou au contraire permettant la mobilité résidentielle dans une conjoncture d'assouplissement de la législation. Rendant possible pour la première fois la résidence permanente et légalisant le regroupement familial, l'immigration reform and control act de 1986 a en particulier eu pour conséquence directe l'accélération de la croissance démographique haïtienne dans le secteur ainsi que la possibilité pour les familles de faire des projets à long terme dans la société d'accueil.

8 À partir de la deuxième moitié des années 1980, la volonté de quitter l'« enclave » haïtienne paupérisée s'est manifestée par l'installation d'un nombre croissant d'originaires d'Haïti dans les banlieues de classes moyennes du nord de la métropole. Les «têtes de ponts » ainsi créées ont permis le délestage d'un quartier ancien saturé. Le déplacement du centre de gravité résidentiel s'est opéré à diverses échelles :

- À l'échelle métropolitaine, il a pris la forme d'un mouvement des quartiers centraux vers les localités de banlieue ;

- À l'échelle des quartiers et localités, il s'est manifesté par des migrations vers les secteurs les plus attractifs du nord et de l'est, comme l'illustre la mobilité résidentielle enregistrée de Buena Vista vers Little River et El Portal ou de North Miami vers North Miami Beach.

La diminution du poids absolu et relatif des Haïtiens dans les quartiers centraux et la constitution de nouveaux «quartiers ethniques» hors de Little Haiti révèlent la complexité croissante d'un territoire de l'immigration en recomposition. De même, la coexistence de deux pôles de concentration démographique haïtienne - l'un au centre, l'autre en périphérie - nuance le schéma traditionnel de regroupement des «minorités ethniques » et des immigrants récents dans les quartiers pauvres des villes centrales. L'expérience résidentielle haïtienne à Miami tend davantage à reproduire celle des Cubains que celle des Noirs états-uniens, du point de vue des formes et de la dynamique de l'insertion.

10 La mobilité résidentielle vers les banlieues reste perçue par les immigrants comme un moyen de promotion sociale et comme une étape hautement symbolique du processus d'insertion dans la société d'accueil. Elle apparaît en même temps comme une stratégie de différenciation vis-à-vis des compatriotes dans une situation sociale plus précaire et n'ayant d'autre alternative que de demeurer dans le «quartier ethnique » originel. La complexité croissante de l'espace d'installation des Haïtiens, caractérisée par une évolution rapide de son étendue et de ses formes, se manifeste par une dichotomie de plus en plus marquée entre l'«enclave» traditionnelle et les nouveaux espaces d'installation en banlieue organisés autour du pôle de North Miami.

11 Cependant, la dilatation et les mutations de cet espace résidentiel ne remettent pas en cause les logiques ségrégatives à la base de l'organisation spatiale métropolitaine. En effet, l'élargissement de l'espace d'installation des immigrés traduit la reproduction en périphérie des logiques de ségrégation - illustrée par le mécanisme de la succession résidentielle - et d'agrégation à l'origine de la constitution d'un quartier haïtien au 
centre de l'agglomération. Les logiques de ségrégation s'avèrent être autant le fruit d'un libre choix que de contraintes discriminatoires, les stratégies des ménages s'opérant dans le cadre d'un marché du logement segmenté sur une base «ethnoraciale ».

Leur insertion spatiale récente s'inscrit donc en continuité avec les modèles d'installation antérieurs et s'inscrit dans le mouvement plus large de l'expansion urbaine des Noirs vers le nord-est de l'espace urbain dans des secteurs antérieurement habités par les classes moyennes blanches. Temporaire à l'échelon du quartier ou de la municipalité, la ségrégation caractérisant l'insertion urbaine des Haïtiens semble s'inscrire dans la durée à l'échelon métropolitain. Les dynamiques de ségrégation et d'agrégation se combinent pour dessiner un territoire où la composante haïtienne représente entre un cinquième et la moitié de la population totale. Ce processus de concentration spatiale à l'œuvre au centre comme en périphérie apparait lourd de conséquences sur les formes de l'appropriation par les immigrés des espaces dans lesquels ils s'installent.

2. De l'insertion à l'appropriation spatiale : Stratégies identitaires haïtiennes et processus de territorialisation

13 Au-delà de la simple territorialisation résidentielle, l'appropriation spatiale revêt ici la triple signification de l'apprentissage de l'espace, de sa prise de possession et de son affectation à une activité déterminée ${ }^{1}$ Au niveau individuel, cette appropriation prend avant tout la forme d'un apprentissage de l'espace par l'immigrant par le biais de représentations et de pratiques territoriales plus ou moins complexes, en fonction de son expérience sociale et de ses référents culturels. L'appropriation mentale (et fonctionnelle) de l'espace mise en œuvre vise à faire sienne la terre d'immigration et à la doter de sens. Les idéologies, les mémoires et les pratiques de l'espace exprimées par les Haïtiens de Miami dévoilent leur rapport intime à leur milieu de vie et renseignent sur la fonction identitaire de leur espace approprié. Les représentations spatiales individuelles révèlent l'affirmation d'un territoire en le différenciant des espaces appropriés par les autres groupes « ethniques » - en particulier de celui des Noirs étatsuniens auxquels les immigrés refusent d'être assimilés ou identifiés. Les repères et références identitaires inscrits dans l'espace par les représentations individuelles fondent le territoire haïtien. Ce territoire, expression spatiale de l'identité du groupe et de son altérité revendiquée, affirme l'existence d'une communauté haïtienne longtemps niée par la société d'accueil. En constituant un espace identifié, familier et reconnu par tous, le territoire perçu s'affirme comme le support spatial de l'insertion sociale du groupe dans la mosaïque métropolitaine.

14 Si les perceptions des individus expriment le lien entre le territoire haïtien et l'altérité du groupe, elles dévoilent en même temps l'hétérogénéité de l'espace approprié en portant l'accent tant sur les oppositions que sur les complémentarités des secteurs le constituant. Quoique les représentations géographiques présentent Little Haiti comme un secteur résidentiel répulsif, un espace d'exclusion, elles le valorisent aussi en tant que territoire de la défense et de la pérennité de la culture du pays d'origine. La dimension culturelle majeure est exprimée par les trajectoires individuelles privilégiant les commerces « ethniques » et les églises de ce quartier (Audebert, 2002). Parallèlement, la fonction résidentielle de la périphérie s'affirme de plus en plus clairement. 
15 Ainsi, les perceptions et pratiques que les Haïtiens ont de leur propre territoire - donc d'eux-mêmes - mettent en relief l'ambivalence du processus d'insertion des immigrés dans la société d'accueil : tout en cherchant à accéder à l'American way of life, synonyme d'un mieux être économique et d'une adhésion aux valeurs dominantes de la société états-unienne, les immigrants restent très attachés à leur culture d'origine, celle qui leur permet de se définir par rapport aux autres. L'appropriation spatiale révélée par les perceptions et pratiques individuelles ne constitue cependant qu'une dimension de l'analyse territoriale. Pour être validé, le schème esquissé par cette " géographicité »pour paraphraser Di Méo - doit être confronté aux dimensions collectives de l'appropriation de l'espace par les Haïtiens à Miami : celles à l'œuvre dans les sphères économique et politique.

16 Au niveau collectif, la « prise de possession » d'une portion de l'aire métropolitaine et son affectation à des activités sociales, commerciales et de représentation politique visent à assurer la pérennité du lien communautaire haïtien en créant de la cohésion, de la solidarité et de la socialité. Le marquage de l'espace constitutif de la prise de possession s'exprimant par rapport à l'Autre, cette pérennité se conçoit également dans la distanciation sociale, culturelle et géographique par rapport aux autres communautés. En effet, la reconnaissance de la population haïtienne comme une composante à part entière de la société floridienne passe par la différenciation sociale et spatiale vis-à-vis de la communauté noire «native» d'une part et des autres populations antillaises d'autre part. L'expérience d'insertion socio-spatiale des Haïtiens tend ainsi à remettre en question à l'échelon métropolitain la logique de l'organisation de la société états-unienne sur la base de catégories "ethniques" artificielles et globalisantes. Paradoxalement, l'intégration de cette population immigrée dans la société multiculturelle états-unienne est donc liée à la mise en exergue de son altérité en vue de défendre ses intérêts propres - ce qui n'empêche toutefois pas les alliances intercommunautaires, la cohabitation spatiale et la genèse de cultures hybrides.

17 Les différentes dimensions de l'intégration se lisent dans le rapport des immigrés à leur espace. Le processus de territorialisation marchande illustre la médiation de l'espace dans l'intégration fonctionnelle des Haïtiens dans la société métropolitaine. Les 485 commerces haïtiens recensés se présentent ici comme des espaces de rencontre, de survie et d'insertion dans la structure économique états-unienne. Mais ils s'affirment aussi comme des lieux hautement symboliques incarnant les représentations collectives issues de l'expérience migratoire, ce que révèle la «territorialisation sémiotique » (photos 3 et 4 ). 
Photo 3 - La $54^{\mathrm{e}}$ rue (Little haiti) centre originel de la vie commerciale et politique des Haïtiens de Miami dans les années 1970 et 1980 .

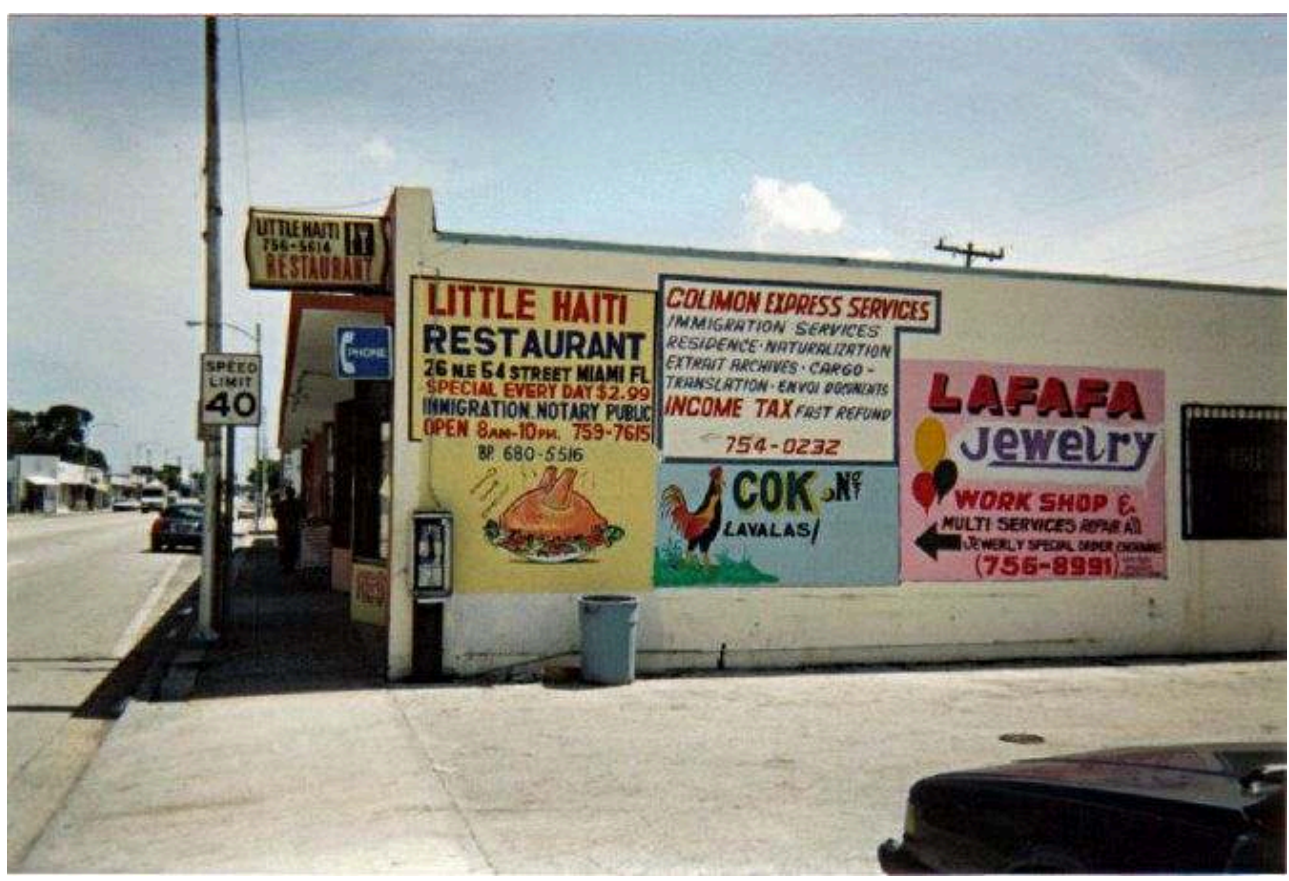

Sur les murs se côtoient publicités commerciales et message politiques. Les restaurants offrent des repas pour 2,99\$... et des services administratifs divers liés à l'immigration.

Photo 4 - Le Haitian Refugee Center (Sant Refijie Ayisyen) sur la $54^{\mathrm{e}}$ rue à Little Haiti.

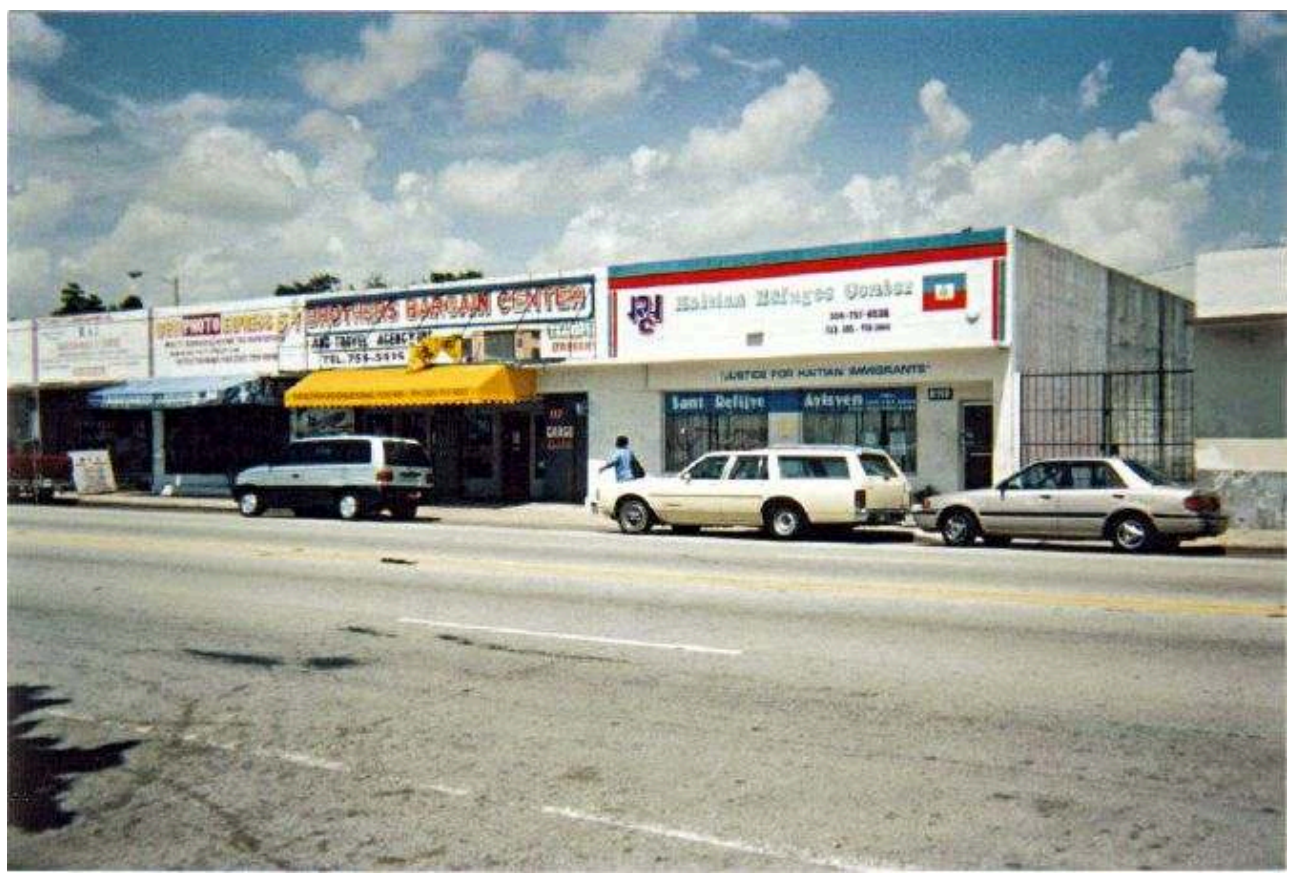

Cette institution est au cœur de la défense des droits des immigrants haïtiens depuis plus de 25 ans Sur sa façade on peut lire : « Justice for Haitian immigrants »

Le marquage du territoire par l'entreprenariat apparaît comme la manifestation la plus visible d'une appropriation du tissu urbain par la communauté haïtienne. L'effectif, la nature et la dynamique spatiale des commerces renseignent sur le lien entre le 
processus d'insertion et celui de territorialisation du groupe. L'appropriation commerciale de l'espace urbain par la communauté traduit bien les modalités de son insertion dans la société d'accueil : si la structuration d'un territoire marchand répond à la nécessité de la population immigrée de reproduire l'environnement social et culturel du pays d'origine dans l'optique d'une adaptation en douceur, elle constitue également un moyen de survie, voire pour certains, de promotion économique. Les immigrants ont donc su utiliser les structures commerciales préexistantes pour accéder à un entreprenariat synonyme de considération sociale dans leur communauté, et pour affirmer leur identité au sein de la société d'accueil (photos 5, 6, 7 et 8).

Photo 5 - L'appropriation symbolique de l'espace par les noms de rue : I'avenue Félix MorisseauLeroy $\left(2^{\mathrm{e}}\right.$ avenue nord-est)

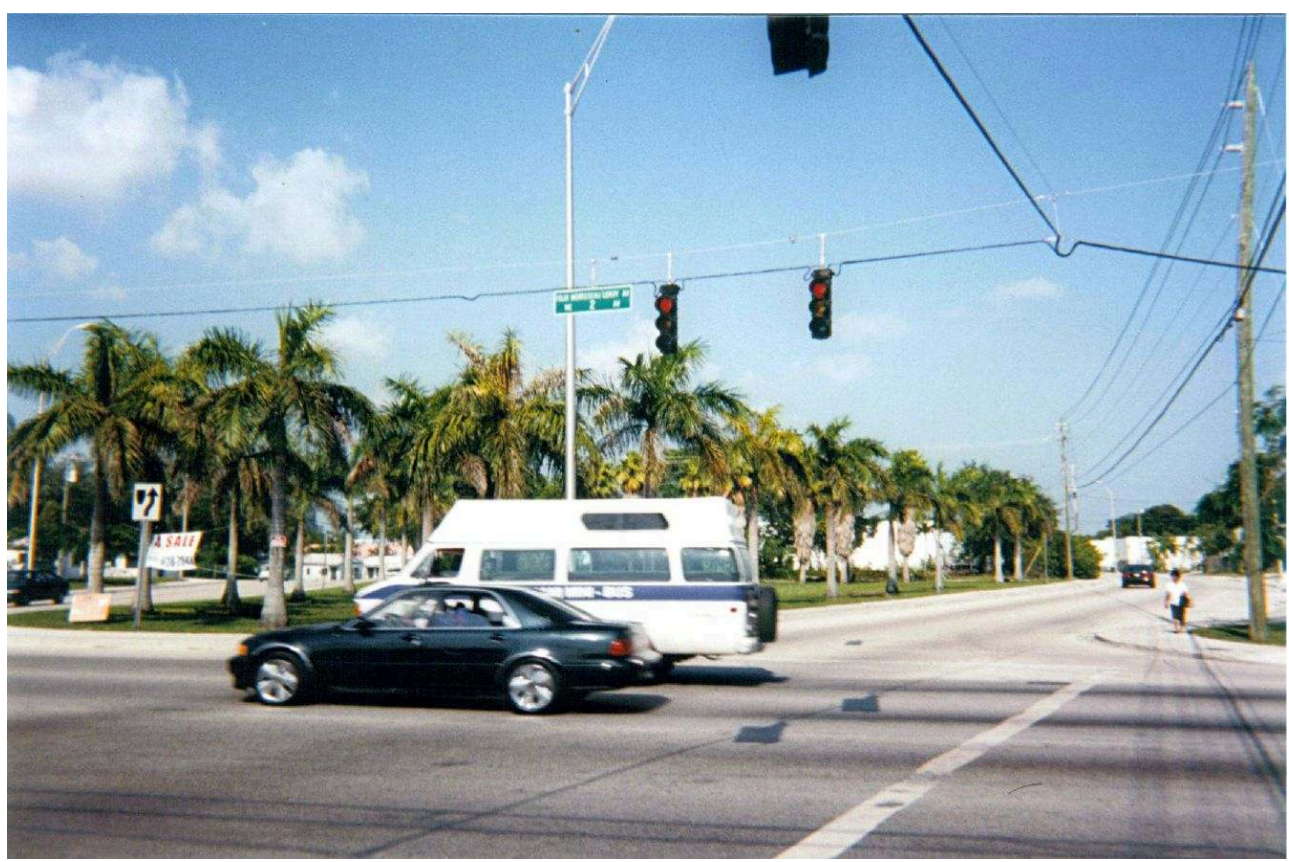

Des modes variés de marquage de l'espace par les Haïtiens 
Photo 6 - L'appropriation symbolique par la peinture : la représentation de l'espace d'un moment fort de l'indépendance d'Haïti sur les murs de Little Haiti.

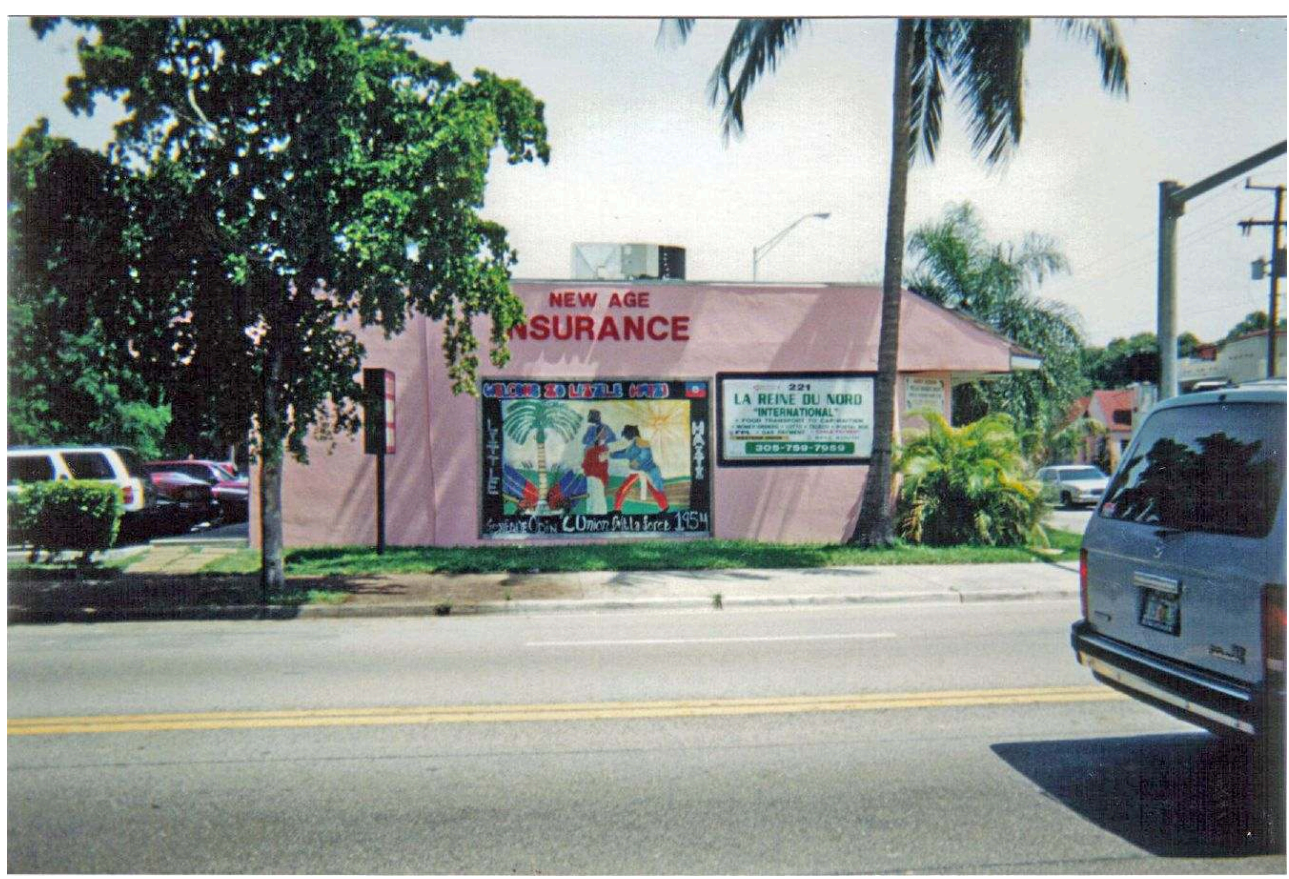

On peut y voir : «Welcome to Little Haîti » ainsi que la devise du pays d'origine «L'union fait la force »

Photo 7 - Le marquage architectural et culturel de Little Haiti et la Librairie Mapou

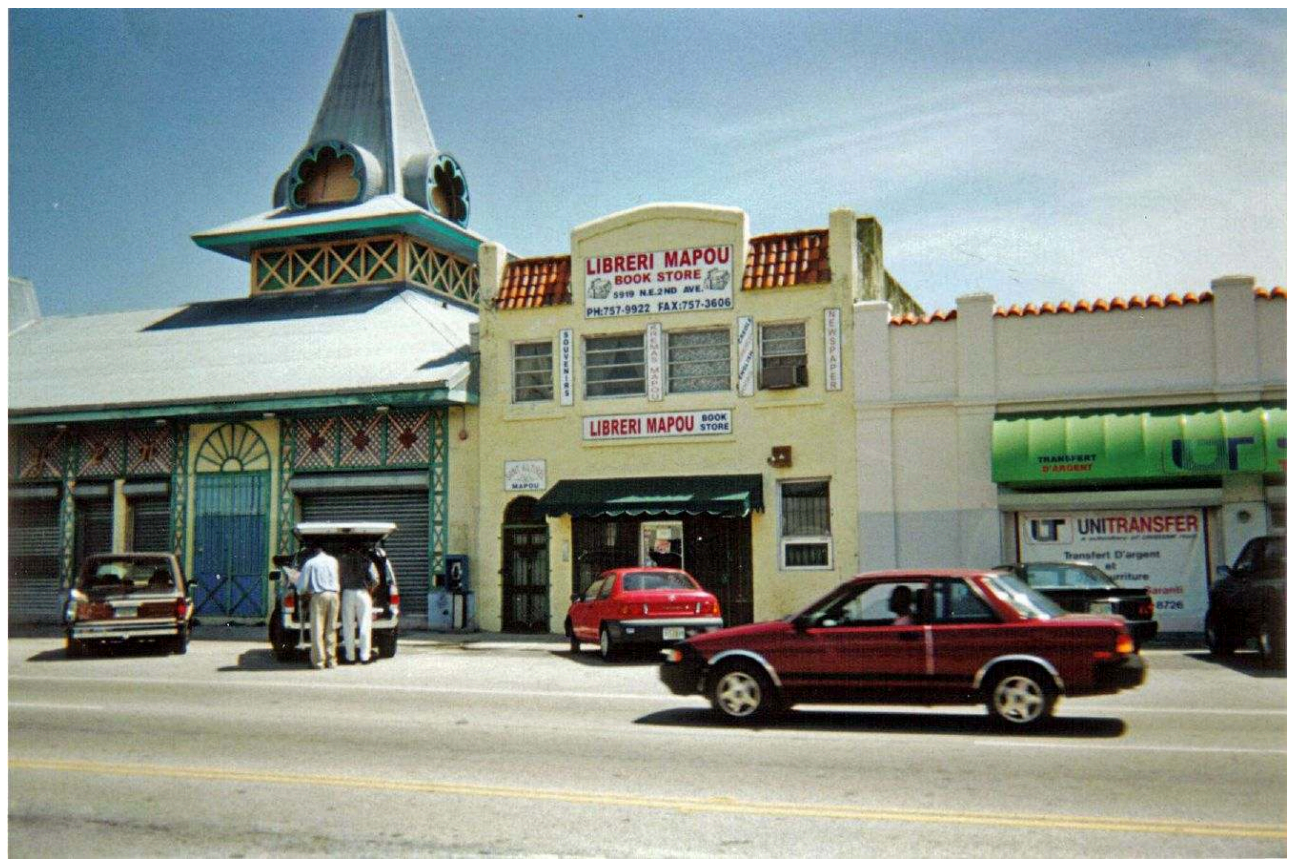

Stratégies identitaires haïtiennes et appropriation commerciale de l'espace 
Photo 8 - Peinture haïtienne sur la façade d'une maison de transfert à Little Haiti

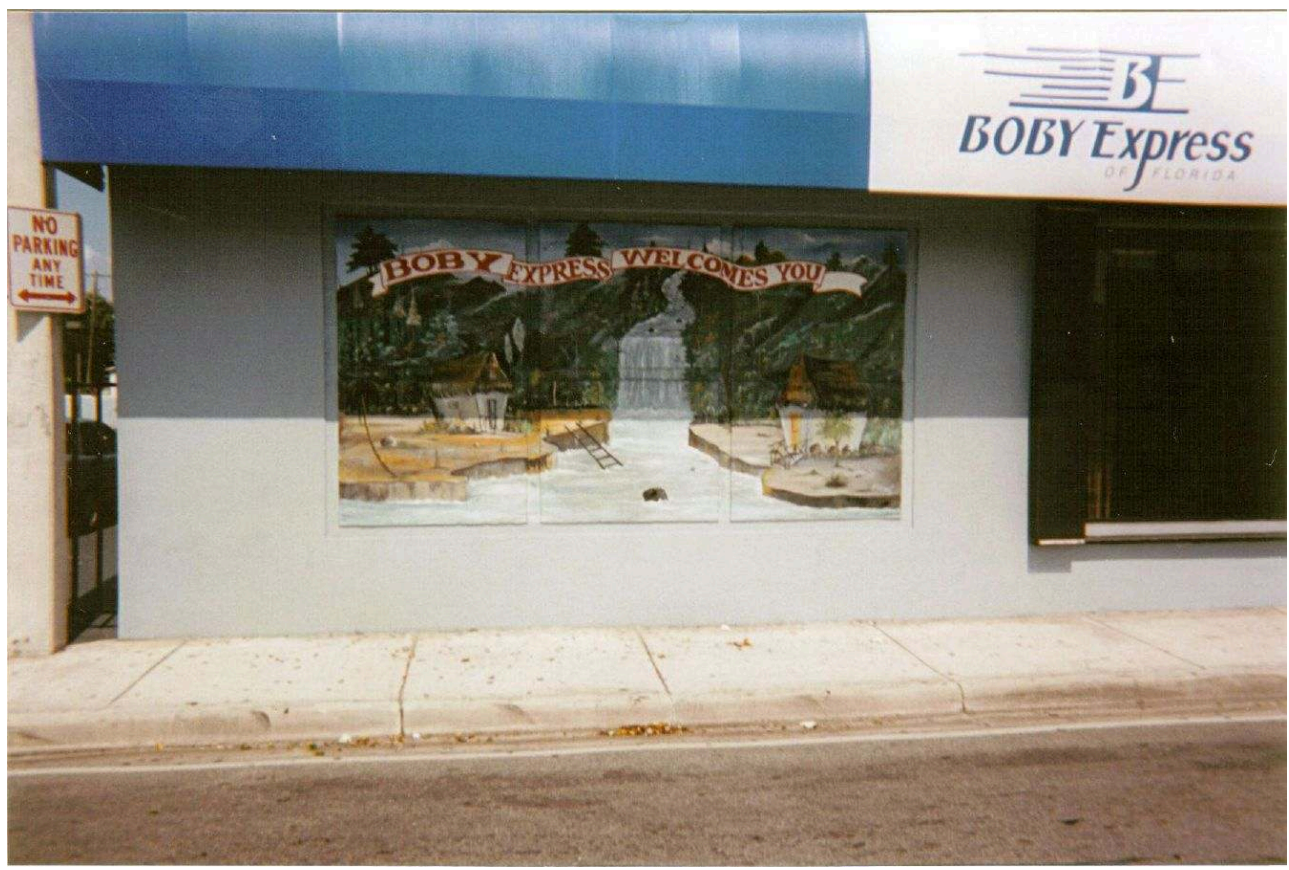

Par les diverses modalités de l'appropriation signifiante dont elle fait l'objet, la territorialisation marchande révèle aussi la pluralité de l'identité (donc des identités) haïtienne(s) en Floride. Les enseignes montrent autant le besoin de maintenir un lien fort avec le pays d'origine, que celui de signifier une identité hybride haïtianoétatsunienne ou l'absence totale de référents culturels haïtiens. L'identité est alors mobilisée comme une stratégie commerciale répondant aux besoins de la population haïtienne ou au contraire visant à élargir le marché à d'autres composantes culturelles de la société d'accueil. L'analyse spatiale met en exergue des relations différenciées entre identité et territorialisation marchande : tandis que le quartier originel reste le lieu où l'affirmation de l'identité du pays d'origine demeure la plus vivace, la reprise de stratégies identitaires autochtones apparaît dominante dans les banlieues. L'expression plus limitée de la différence culturelle dans les secteurs d'implantation récente est liée à la diversité des commerces, dont les produits et services s'adressent à une clientèle plus hétérogène.

Si l'identité haïtienne exprimée ainsi apparaît davantage fondée sur une représentativité symbolique de la présence du groupe, d'autres formes d'appropriation spatiale liées à l'expression identitaire collective ont été mises en œuvre par les Haïtiens de Miami, prenant la forme d'une représentativité institutionnelle. De ce point de vue, l'analyse du lien entre l'intégration participative des immigrés - phase la plus récente de leur processus d'insertion en Floride - et leur appropriation politique de l'espace urbain apparaît riche d'enseignements.

21 La territorialisation politique marque l'intégration participative des Haïtiens qui consolide le double sentiment d'appartenance à la communauté haïtienne et à la communauté des citoyens de la société d'accueil. L'apprentissage du système politique états-unien par les Haïtiens apparaît comme un élément fondamental de la cohésion de cette communauté d'immigrés, qui lui permet d'affirmer avec davantage de force son existence en tant qu'entité «ethnique ». Son apparition sur la scène politique de la société d'accueil s'exprime à travers une remise en cause de la nature des relations que 
les Haïtiens entretenaient jusqu'ici avec les autres populations locales. Les Cubains, dont le succès politique a été favorisé par la faible organisation des Noirs états-uniens, leur marginalisation sociale et par le repli progressif des Blancs non hispaniques, prennent actuellement conscience de l'émergence d'un «bloc » électoral haïtien qu'ils convoitent de plus en plus. De leur côté, les Haïtiano-étatsuniens rêvent de reproduire le modèle d'ascension politique de la communauté cubaine. Ils souhaitent faire de North Miami un bastion leur servant de base à l'élargissement de leur force politique, à l'instar du rôle que la municipalité d'Hialeah (voir carte) a joué pour les Cubains il y a deux décennies.

L'apparition des Haïtiens sur la scène politique de Miami tend à crisper la communauté noire "native » qui souhaitait utiliser le potentiel électoral croissant des immigrés pour servir ses propres intérêts et sortir de sa marginalisation sur le plan local. Intimement liée à l'importance que les noirs autochtones accordent à la solidarité de couleur, leur sensibilité à la cause haïtienne est indéniable. Cependant, leur combat est souvent apparu trop différent des aspirations d'une population immigrante dont ils ne partagent ni la langue, ni la culture. De manière générale, l'expérience haïtienne à Miami constitue une remise en cause du modèle politique de la société d'accueil organisé en cinq catégories "ethno-raciales" très simplificatrices (Noirs, Blancs, Hispaniques, Asiatiques, Amérindiens) et peu pertinentes pour appréhender une réalité culturelle et sociale états-unienne de plus en plus complexe. Elle revêt la même signification que celles de l'émergence des «blocs » politiques irlandais à Boston et juif à New York il y a un siècle, et de l'apparition d'un « bloc » électoral cubain à Miami plus récemment. L'accession à la visibilité politique des Haïtiens s'opère par la prise de distance vis-à-vis de catégories « ethniques » trop larges pour défendre leurs intérêts particuliers.

Conclusion

23 Dans les champs résidentiel, commercial et politique, l'appropriation d'une partie de l'espace métropolitain par la communauté haïtienne s'est opérée dans les secteurs périphériques de l'agglomération, synonymes d'une meilleure intégration. A North Miami, l'insertion spatiale revêt en effet une autre dimension: en s'appropriant l'espace politique et marchand de cette municipalité de banlieue, les immigrés ne revendiquent plus seulement le droit d'exister et d'être reconnus en tant que communauté à part entière dans la société d'accueil, mais aussi le droit de participer aux affaires de la cité et de maîtriser leur propre destin. L'appropriation commerciale et politique de l'espace constitue la marque territoriale d'un véritable processus d'intégration des Haïtiens, au sens double de l'interaction et de la participation.

La banlieue Nord de Miami apparaît en effet comme le théâtre d'une interaction de plus en plus poussée avec les autres communautés de la société d'accueil, à la faveur d'une cohabitation ethno-résidentielle plus marquée. Mais l'intégration se lit aussi dans une participation croissante des Haïtiens en tant que communauté ethnique à la vie culturelle et politique locale. Cependant, l'identité du pays d'origine - enrichie d'expériences et d'apports culturels états-uniens - étant mobilisée à chaque étape du processus d'interaction/participation dans un contexte socio-institutionnel de valorisation de la différence et de la construction ethnique, cette intégration progressive et bien comprise ne s'accompagne pas forcément d'une assimilation à la culture dominante de la société d'installation. 


\section{BIBLIOGRAPHIE}

\section{Références bibliographiques}

Audebert Cédric, 2004.Immigration et insertion urbaine en Floride : le rôle de la famille transnationale haïtienne. Revue Européenne des Migrations Internationales, vol. 20, n 3, p. 127-146.

Audebert Cédric, 2002. Le fait religieux dans l'insertion et l'organisation spatiale de la communauté haïtienne de Miami. Géographie et Cultures, n 43, p. 107-127.

Boswell Thomas D., 1994. The Cubanization and Hispanicization of Metropolitan Miami. Cuban American Policy Center, Miami, 46 p.

Boswell Thomas D., Skop Emily, 1995. Hispanic National Groups in Metropolitan Miami. Cuban American Policy Center, Miami, 57 p.

Catanese Anthony V., 1999. Haitians : Migration and Diaspora. Westview Press, Boulder (Colorado), $256 \mathrm{p}$.

Grenier Guillermo, Stepick Alex (dir.), 1992. Miami Now! Immigration, Ethnicity and Social Change. University Press of Florida, Gainesville, 219 p.

Glick Schiller Nina, Fouron Georges E., 1999. Terrain of blood and nation : Haitian transnational social fields. Ethnic and Racial Studies, vol. 22, n 2, p. 340-366.

Icart Jean-Claude, 1987. Négriers d'eux-mêmes : Essai sur les Boat People haïtiens en Floride. CIDIHCA, Montréal, 188 p.

Laguerre Michel S., 1984. American odyssey: Haitians in New York City. Cornell University Press, Ithaca, N.Y., 198 p.

Mittelberg David, Waters Mary, 1992.The process of ethnogenesis among Haitian and Israeli immigrants in the United States. Ethnic and Racial Studies, vol. 15, n 3, p. 412-435.

Portes Alejandro, Stepick Alex, 1993. City on the Edge : the transformation of Miami. University of California Press, Berkeley, 281 p.

Stepick Alex, 1998. Pride against prejudice : Haitians in the United States. Allyn and Bacon, Boston, $134 \mathrm{p}$.

Stepick Alex, 1992. The refugees nobody wants : Haitians in Miami. In Grenier Guillermo et Stepick Alex, (dir.). Miami Now! Immigration, Ethnicity and Social Change. University Press of Florida, Gainesville, p. 57-80.

\section{NOTES}

1. Selon la typologie de Brunet dans son dictionnaire Les Mots de la géographie de 1990. 


\section{RÉSUMÉS}

Le schéma migratoire haïtien s'est traduit par la genèse d'un champ migratoire international entre le pays d'origine et Miami, où la population d'origine haïtienne a enregistré une croissance rapide. Dans une métropole floridienne marquée par une forte ségrégation « ethno-raciale » et socio-économique, les nouveaux venus se sont installés à l'origine dans les quartiers noirs centraux paupérisés. Les incidences de la politique migratoire fédérale sont apparues contrastées, occasionnant le repli sur soi des immigrants à Little Haiti lorsqu'elle était défavorable ou rendant possible la mobilité résidentielle dans une conjoncture d'assouplissement de la législation. Ultérieurement, la complexité croissante de l'espace de l'immigration haïtienne, caractérisé par une évolution rapide de son étendue et de ses formes, s'est manifestée par une dichotomie marquée entre l'« enclave " traditionnelle et les nouveaux espaces d'installation en banlieue. Résultat d'une dynamique d'agrégation autant choisie que contrainte, la concentration spatiale des Haïtiens à Miami s'est traduite par la genèse de secteurs d'implantation privilégiée, terrains favorables à la mise en place de processus de territorialisation complexes. L'apprentissage mental et fonctionnel de l'espace vise à faire sienne la terre d'immigration et à la doter de sens, et apparaît comme le préalable à la mise en place de stratégies collectives de territorialisation. La "prise de possession" d'une portion de l'aire métropolitaine et son affectation à des activités sociales, commerciales et de représentation politique visent à assurer la pérennité du lien communautaire haïtien en créant de la cohésion, de la solidarité et de la socialité.

Haitian emigration has evolved within the growing dependency of Haiti from the United States throughout the $\mathrm{XX}^{\text {th }}$ century. The Haitian migratory pattern took on the form of an international migratory field linking the source country with Miami, where the population from Haitian ancestry has recorded a rapid growth. In a Floridian metropolis branded with a strong « ethnoracial » and economic segregation, the newcomers originally settled in the impoverished Black inner city neighborhoods. The effects of the federal immigration policy has been contrasted, bringing about the withdrawal of immigrants into themselves in Little Haiti when the policy was unpropitious or allowing a residential mobility in times of relaxed legislation. Subsequently, the growing complexity of the Haitian immigration space, characterized by a rapid extension and change in its forms, is apparent through a dichotomy between the traditional enclave and the new suburban settlements. Resulting from an aggregation/segregation dynamics, Haitian spatial concentration in Miami found expression in particular settlement locations, where processes of territorialization have taken place. The mental and functional adaptation to the place of settlement aims at appropriating it by giving it a sense of belonging, and appears to be the first step to collective strategies of territorialization. The take over of a section of the metropolitan area and its affectation to social, commercial and political forms of representation aim at perpetuating the Haitian community's bond by creating cohesion, solidarity and sociality.

\section{INDEX}

Keywords : immigration, insertion, Miami, territory, Haitians, United States, Caribbean, metropolization, identity, community

Mots-clés : territoire, Haïtiens, Etats-Unis, Caraïbe, ségrégation, métropolisation, identité, communauté 


\section{AUTEUR}

\section{CÉDRIC AUDEBERT}

Audebert Cédric [cedric.audebert@univ-poitiers.fr] est chargé de recherche au CNRS, dans le laboratoire Migrinter (UMR 6588) Il est, entre autres, responsable scientifique de la revue e-migrinter, membre fondateur du bureau scientifique du centre international de recherches sur les esclavages (EHESS/CNRS). Il a publié une vingtaine d'articles sur le thème des migrations internationales issues de la Caraïbe et deux ouvrages, dont L'insertion socio-spatiale des Haïtiens à Miami (L'Harmattan, coll. Populations, 2006). 